\title{
Joint Sensor Location/Power Rating Optimization for Temporally-Correlated Source Estimation
}

\author{
Osama M. Bushnaq, Anas Chaaban and Tareq Al-Naffouri \\ Electrical Engineering department, King Abdullah University of Science and Technology, \\ Thuwal, Saudi Arabia, e-mails: \{osama.bushnaq, anas.chaaban, tareq.alnaffouri\}@kaust.edu.sa
}

\begin{abstract}
The optimal sensor selection for scalar state parameter estimation in wireless sensor networks is studied in the paper. A subset of $N$ candidate sensing locations is selected to measure a state parameter and send the observation to a fusion center via wireless AWGN channel. In addition to selecting the optimal sensing location, the sensor type to be placed in these locations is selected from a pool of $T$ sensor types such that different sensor types have different power ratings and costs. The sensor transmission power is limited based on the amount of energy harvested at the sensing location and the type of the sensor. The Kalman filter is used to efficiently obtain the MMSE estimator at the fusion center. Sensors are selected such that the MMSE estimator error is minimized subject to a prescribed system budget. This goal is achieved using convex relaxation and greedy algorithm approaches.
\end{abstract}

Index Terms-Wireless sensor networks, sensor selection, convex optimization, Kalman filter, estimation.

\section{INTRODUCTION}

Wireless sensor networks (WSN) have been gaining increasing interest in the last few years due to their role in emerging technologies such as Internet of things (IoT). Despite the wide range of studies regarding WSN optimization in the literature, there still are many challenges in implementing these networks. Sensors are expected to harvest energy and control their consumptions to have self powered sensing nodes and, on the other hand, obtain a reliable and accurate measurements, which are affected by observation and communication noises. The complexity of such problems lies in designing a mathematical model that accounts for many factors. Such a model might result in minimizing non-convex nor sub-modular functions over a large number of sensors.

Two related, yet different, major challenges in sensor networks are, (1) to select a set of sensors with suitable specifications and place them in strategic locations at the design time (offline sensor selection) [1]-[6], and, (2) to schedule sensors operation at the running time. [7]-[10].

In [2]-[4], [7], [11], a static measurements model was considered such that the distributed parameter estimation is minimized based on the current measurement statistics. These works considered a source without temporal correlation. In [2] the sensor placement via convex relaxation approach was introduced for static state estimation. In [4], [11], the same problem was solved taking into account the amount of $\mathrm{EH}$

This work is supported by the KAUST-MIT-TUD consortium under grant OSR-2015-Sensors-2700. at the sensor nodes and the wireless communication channel quality to a fusion center.

Nevertheless, physical quantities in nature tend to change slowly over time. Hence, temporal correlation between observations that are separated by orders of few seconds tend to be high. Ignoring such correlation will significantly degrade the estimation performance for a wide range of applications. The first order Gauss-Markov process is a simple and popular model that is used to capture the dynamics of the unknown parameter. The MMSE estimator and its error can be efficiently obtained through the Kalman filter [12]. In [8], [13] the MMSE estimation error is minimized over only a finite time horizon.

Since exhaustive search for the optimal sensor placement is clearly intractable for large sensor networks, other approaches such as convex relaxation and greedy algorithms are popular. In general, convex optimization tools are avoided in the vectorstate estimation problems due to the difficulty of dealing with the discrete algebraic Riccati equation (DARE), which arises from solving for the MMSE Kalman estimation error covariance matrix. It is shown in [6], [8], [14] that the objective function of the sensor selection/scheduling problems for linear dynamical process estimation are in general, not submodular or supermodular. Therefore the greedy algorithms performance is not guaranteed. Scalar state estimation is studied in [15], [9] in order to obtain a simple and optimal solution.

In [1], [5], [6], [14] several greedy algorithms were proposed to minimize the MMSE estimation error for the vector state linear dynamical system subject to a prescribed number of sensing nodes. However, non of these studies has considered the cost of sending the sensors observations to a fusion center. In [15], sensors are selected for optimal scalar state dynamical system estimation at a remote estimator. The authors assume uncertainty of a successful reception at the estimator based on the sensor transmission power and assume that the sensors receive feedback upon successful transmissions.

In spite of the general awareness of the energy resources scarcity in WSNs, only few studies allocate the sensor transmission power rating for dynamic estimation. In [15], the sensor selection problem is solved such that either a low power sensor or a high power sensor is placed at each candidate sensing node.

The main goal of this paper is to improve the offline sensor selection by considering different practical issues to guarantee a better overall system performance in the sense of minimizing the MMSE estimator error at a central processing unit. We 
focus on the scalar state estimation and show that for the problem of fixed power rating sensor placement, the solution can be obtained by ordering the contribution of each sensor, even while considering some practical issues such as the amount of EH and the AWGN channel quality. Moreover, we generalize the approach in [15] by considering the availability of $T$ sensor types each with some power rating and cost to be placed at the candidate sensing node. Since the cost of the battery and the EH equipments are relatively expensive, such a model will optimize the budget efficiency by placing an expensive sensor type (with higher power rating) at strategic locations and a cheaper sensor type (with less power rating) at other candidate sensing locations. The contribution of this paper can be summarized as follows:

1) This work is the first work to consider state estimation for a linear dynamical system taking into account the amount of $\mathrm{EH}$ and the AWGN channel quality.

2) In addition to sensor placement, sensors are selected from a pool of available sensor types such that each sensor type have some power rating and cost.

3) We present a sub optimal solution via convex relaxation. Further, a dynamic program is proposed to optimally solve the selection problem. We show that the problem is similar to the $0-1$ knapsack problem with extra restriction.

\section{SYSTEM MODEL}

Consider a scalar dynamical system model for the state parameter $x[n]$, which is described by the first order GaussMarkov process,

$$
x[k]=a x[k-1]+\omega[k], \quad k>0
$$

where $\mathbb{E}\{x[0]\}=\mu_{s}$ and $\omega[k] \sim \mathcal{N}\left(0, \sigma_{\omega}^{2}\right)$ is the driving or excitation noise. We assume that $\mu_{s}$ and $\omega[k]$ are independent and, $\omega\left[k_{1}\right]$ and $\omega\left[k_{2}\right]$ are uncorrelated $\forall k_{1} \neq k_{2}$. For a stabilizable $\left(a, \sigma_{\omega}\right)$ and as $k \rightarrow \infty, \mu_{x}=\lim _{k \rightarrow \infty} \mathbb{E}\{x[k]\}=0$ and $\sigma_{x}^{2}=\lim _{k \rightarrow \infty} \operatorname{Var}(x[k])=\sigma_{\omega}^{2} /\left(1-a^{2}\right)$.

We would like to design the WSN to accurately estimate $x[k]$ subject to a prescribed system budget. Consider a set of $N$ candidate sensing locations. The observed measurements at these locations are given by the linear model,

$$
\mathbf{y}[k]=\mathbf{c} x[k]+\boldsymbol{\nu}[k],
$$

such that the $n$-th element of $\mathbf{y}[k]$ represents the observation at the $n$-th sensing location, $\forall n \in\{1, \ldots, N\}, \mathbf{c}=\left[c_{1}, \ldots, c_{N}\right]^{T}$ is the state measurement vector which is assumed to be known and $\nu[k]=\left[\nu_{1}[k], \ldots, \nu_{N}[k]\right]^{T}$ is the measurement noise with $\nu_{n}[k] \sim \mathcal{N}\left(0, \sigma_{\nu}^{2}\right), \forall n \in 1, \ldots, N$.

Sensors are assumed to be self-powered through collecting energy from different resources in the environment such as EM waves, solar energy, and temperature fluctuations. Therefore, the average available energy for harvesting is varying over different sensing locations. The system designer has the option to select a sensor type from a pool of $T$ available sensor types. Sensors with more expensive types are equipped with more energy harvesting equipments and have higher battery capacities, therefore can harvest energy more efficiently. We denote this efficiency by $\eta_{t}$. We define $\boldsymbol{w}_{n}^{T}=\left[w_{n, 1}, \ldots, w_{n, T}\right]$ as the sensor type selection vector at the $n$-th sensing location, where $w_{n, t}=1$ indicates that sensor type $t$ is placed at location $n$, and $w_{n, t}=0$ otherwise.

Remark 1: We restrict the number of selected sensors at any location to one sensor at most, i.e., $\left|\boldsymbol{w}_{n}^{T}\right|=1$ if a sensor of type $t, \forall t \in\{1, \ldots, T\}$ is placed at the $n$-th sensing location or $\left|\boldsymbol{w}_{n}^{T}\right|=0$ if no sensor is placed at the $n$-th sensing location.

Consequently, the sensor transmission power at the $n$-th sensing location is a function of the available energy at the $n$-th sensing location and the EH efficiency of the selected sensor type at that location. To be more specific, available the transmission power will be

$$
p_{n}\left(\boldsymbol{w}_{n}^{T}\right)=f\left(\rho_{n}, \boldsymbol{w}_{n}^{T} \boldsymbol{\eta}\right),
$$

such that $\rho_{n}$ is the average power available at the $n$-th sensing location and $\boldsymbol{w}_{n}^{T} \boldsymbol{\eta}$ is the EH efficiency of the selected sensor type where $\boldsymbol{\eta}=\left[\eta_{1}, \ldots, \eta_{T}\right]^{T}$.

Remark 2: Clearly, $f\left(\rho_{n}, 0\right)=0$. An exemplary $f\left(\rho_{n}, \boldsymbol{w}_{n}^{T} \boldsymbol{\eta}\right)$ is the function, $\min \left(\rho_{n} \boldsymbol{w}_{n}^{T} \boldsymbol{\eta}, m \boldsymbol{w}_{n}^{T} \boldsymbol{\eta}\right)$, where $m$ is a positive constant representing an upper limit for $\mathrm{EH}$.

Selected sensors send their observations through orthogonal wireless channels to a fusion center. Assuming an AWGN channel with deterministic gain, $h_{n}$, and receiver noise, $u_{n}[k] \sim \mathcal{N}\left(0, \sigma_{u}^{2}\right), \forall n \in[1, \ldots, N]$, the received signal at the fusion center is formulated as,

$$
\mathbf{z}(\mathbf{W})[k]=\mathbf{G}(\mathbf{W}) \mathbf{y}[k]+\left[\left|\boldsymbol{w}_{1}^{T}\right|, \ldots,\left|\boldsymbol{w}_{N}^{T}\right|\right]^{T} \circ \mathbf{u}[k]
$$

where $\mathbf{W} \in \mathbb{R}^{N \times T}$ is the selection matrix such that $\boldsymbol{w}_{n}^{T}$ is its $n$-th row, $\circ$ is the Hadamard product, $\mathbf{u}[k]=\left[u_{1}[k], \ldots, u_{N}[k]\right]^{T}$ and $\mathbf{G}(\mathbf{W})=$ $\operatorname{diag}\left(g_{1}\left(\boldsymbol{w}_{1}^{T}\right), \ldots, g_{N}\left(\boldsymbol{w}_{N}^{T}\right)\right)$, such that

$$
g_{n}\left(\boldsymbol{w}_{n}^{T}\right)=h_{n} \sqrt{\frac{p_{n}\left(\boldsymbol{w}_{n}^{T}\right)}{\mathbb{E}\left\{y_{n}^{2}[k]\right\}}} .
$$

Note that the observation is scaled by the observation energy, $\mathbb{E}\left\{y_{n}^{2}[k]\right\}=c_{n}^{2} \sigma_{x}^{2}+\sigma_{\nu}^{2}$, to force the transmission power to $p_{n}\left(\boldsymbol{w}_{n}^{T}\right) . \mathbf{z}(\mathbf{W})[k]$ can be rewritten as,

$$
\mathbf{z}(\mathbf{W})[k]=\tilde{\mathbf{c}}(\mathbf{W}) x[k]+\tilde{\boldsymbol{\nu}}(\mathbf{W})[k]
$$

where

$$
\begin{aligned}
\tilde{\mathbf{c}}(\mathbf{W}) & =\mathbf{G}(\mathbf{W}) \mathbf{c} \\
\tilde{\boldsymbol{\nu}}(\mathbf{W})[k] & =\mathbf{G}(\mathbf{W}) \boldsymbol{\nu}[k]+\left[\left|\boldsymbol{w}_{1}^{T}\right|, \ldots,\left|\boldsymbol{w}_{N}^{T}\right|\right]^{T} \circ \mathbf{u}[k]
\end{aligned}
$$

$\tilde{\boldsymbol{\nu}}(\mathbf{W})[k]$ is a Gaussian random vector with zero mean and a diagonal covariance matrix $\Sigma(\mathbf{W})$, such that its $n$-th element,

$$
\Sigma_{n, n}(\mathbf{W})=g_{n}^{2}\left(\boldsymbol{w}_{1}^{T}\right) \sigma_{\nu}^{2}+\left|\boldsymbol{w}_{n}^{T}\right|^{2} \sigma_{u}^{2}
$$

It is assumed that $a, \sigma_{\omega}^{2}, \mathbf{c}, \sigma_{\nu}^{2}, h_{n}$ and $\sigma_{u}^{2}$ are known at the fusion center. We further assume that the fusion center has the statistics of the average $\mathrm{EH}$ at each sensor location and type. The task of the fusion center is to estimate $x[k]$ with minimal MMSE error. This is described next. 


\section{Problem Statement}

Given $N$ candidate sensing locations and $T$ sensor types with different $\mathrm{EH}$ capabilities, battery capacities and prices, we would like to jointly choose the optimal sensor locations and types that minimize the estimation error subject to a threshold on the system budget. Assuming that $\left(a, \sigma_{\omega}\right)$ is stabilizable, the MMSE Kalman prediction error and the MMSE Kalman estimation error converge to [16], [17]

$$
\begin{aligned}
& M_{p}(\mathbf{W})=a^{2} M(\mathbf{W})+\sigma_{\omega}^{2} \\
& M(\mathbf{W})=\left[1-\tilde{\mathbf{c}}^{T}\left(\frac{1}{\left(a^{2} M(\mathbf{W})+\sigma_{\omega}^{2}\right)} \boldsymbol{\Sigma}+\tilde{\mathbf{c}} \tilde{\mathbf{c}}^{T}\right)^{-1} \tilde{\mathbf{c}}^{T}\right] \\
&\left(a^{2} M(\mathbf{W})+\sigma_{\omega}^{2}\right)
\end{aligned}
$$

as $k \rightarrow \infty$, respectively. These errors are caused by the noisy measurements at the sensor level and the receiver noise at the fusion center.

By defining $\lambda$ as the system total budget and $\boldsymbol{\beta}=$ $\left[\begin{array}{lll}\beta_{1} & \ldots & \beta_{T}\end{array}\right]^{T}$ as a cost vector such that $\beta_{t}$ corresponds to the given price of sensor type $t, \forall t \in\{0, \ldots, T\}$, the MMSE Kalman estimation error is minimized by solving the optimization problem:

$$
\begin{array}{lll}
\min _{\left\{w_{n, t}\right\}} & M(\mathbf{W}) \\
\text { subject to } & \sum_{n=1}^{N} \boldsymbol{w}_{n}^{T} \boldsymbol{\beta} \leq \lambda & \\
& w_{n, t} \in\{0,1\}, \quad \forall n, t \\
& \left|\boldsymbol{w}_{n}^{T}\right| \in\{0,1\}, \quad \forall n,
\end{array}
$$

Recall that the element $w_{n, t}$ is equal to 1 if the sensor at location $n$ and type $t$ is selected, otherwise, $w_{n, t}=0$. Neither the objective function in (12) nor the constraints (12b) and (12c) are convex. Hence, The optimization problem cannot be efficiently solved using well known methods [18].

\section{SENSOR SELECTION}

We propose two solutions for the problem defined in (12). One solution is obtained through relaxing the problem to a convex optimization problem. The other solution is obtained by greedy sensor selection.

\section{A. Convex optimization relaxation}

The expression of the Kalman MMSE estimation error in (11) is not convex. To simplify (11), the matrix inversion lemma is used. Therefore, the Kalman estimation error is rewritten as,

$$
\begin{aligned}
M(\mathbf{W}) & =\left(1-\left[1+\tilde{\mathbf{c}}^{T}\left(a^{2} M(\mathbf{W})+\sigma_{\omega}^{2}\right) \boldsymbol{\Sigma}^{-1} \tilde{\mathbf{c}}\right]^{-1}\right. \\
& \left.\left(a^{2} M(\mathbf{W})+\sigma_{\omega}^{2}\right) \boldsymbol{\Sigma}^{-1} \tilde{\mathbf{c}}\right)\left(a^{2} M(\mathbf{W})+\sigma_{\omega}^{2}\right) \\
& =\frac{\left(a^{2} M(\mathbf{W})+\sigma_{\omega}^{2}\right)}{1+\left(a^{2} M(\mathbf{W})+\sigma_{\omega}^{2}\right) \tilde{\mathbf{c}}^{T} \boldsymbol{\Sigma}^{-1} \tilde{\mathbf{c}}}
\end{aligned}
$$

Letting $\alpha=\frac{1}{\tilde{\mathbf{c}}^{T} \boldsymbol{\Sigma}^{-1} \tilde{\mathbf{c}}}$, (13) is reformulated as,

$$
\frac{a^{2} M(\mathbf{W})^{2}}{\alpha}+\left(1+\frac{\sigma_{\omega}^{2}}{\alpha}-a^{2}\right) M(\mathbf{W})-\sigma_{\omega}^{2}=0
$$

and therefore,

$$
\begin{aligned}
M(\mathbf{W}) & =\frac{\sqrt{\left(1-a^{2}\right)^{2} \alpha^{2}+\left[2\left(1-a^{2}\right) \sigma_{\omega}^{2}+4 a^{2} \sigma_{\omega}^{2}\right] \alpha+\sigma_{\omega}^{4}}}{\left(2 a^{2}\right)} \\
& -\left(\left(1-a^{2}\right) \alpha+\sigma_{\omega}^{2}\right) /\left(2 a^{2}\right)
\end{aligned}
$$

As $\alpha$ increases, we note that the first term of the right hand side is increasing faster than the decrease in the second term in (14). Therefore, the error $M(\mathbf{W})$ is monotonically increasing with the increase of $\alpha$. The minimizer of $M(\mathbf{W})$ in terms of $\alpha$ is $\alpha=0$ which results in error $M(\mathbf{W})=0 . \alpha$ is always greater than zero by definition. Hence, minimizing $M(\mathbf{W})$ is equivalent to minimizing $\alpha$. Therefore, we aim to select a subset of sensor locations and types such that $\alpha$ is minimized under the problem constraints defined in (12). Since $w_{n, T} \in$ $\{0,1\}$ and $\left|\boldsymbol{w}_{n}^{T}\right| \in\{0,1\}$, it is not hard to show from (7) and (9) that,

$$
\begin{aligned}
\alpha & =\frac{1}{\sum_{n=1}^{N} \tilde{c}_{n}^{2} / \Sigma_{n, n}} \\
\frac{1}{\alpha} & =\sum_{n=1}^{N} \frac{c_{n}^{2}}{\sigma_{\nu}^{2}} \cdot\left[1-\frac{\sigma_{u}^{2}}{g_{n}^{2}\left(\boldsymbol{w}_{n}^{T}\right) \sigma_{\nu}^{2}+\sigma_{u}^{2}}\right]
\end{aligned}
$$

where $g_{n}\left(\boldsymbol{w}_{n}^{T}\right)$ is as defined in (5).

Maximizing $1 / \alpha$ is equivalent to minimizing $\alpha$, hence equivalent to minimizing $M(\mathbf{W})$. From (15), it is clear that $1 / \alpha$ is concave function of the selection matrix $\mathbf{W}$. Therefore, it is easily maximized subject to convex constraints. However, the constraints in (12) are non-convex due to the non-convexity of the constraints (12b) and (12c). Relaxing (12) to the following problem,

$$
\begin{array}{ll}
\max _{\left\{w_{n, t}\right\}} & \frac{1}{\alpha} \\
\text { subject to } & \sum_{n=1}^{N} \boldsymbol{w}_{n}^{T} \boldsymbol{\beta} \leq \lambda \\
& w_{n, t} \in[0,1], \quad \forall n, t \\
& \boldsymbol{w}_{n}^{T} \mathbf{1} \in[0,1], \quad \forall n,
\end{array}
$$

results in an MMSE Kalman estimation error which can be used as a lower bound for the actual error.

Because of the relaxation of the binary constraints (16b) and (16c), the obtained solution is, in general, not binary as required. A rounding algorithm is needed in order to maintain a binary solution. Some of these algorithms are explained in [2], [3]. The used rounding algorithm in this paper is modified to jointly round sensors' locations and types, (details are omitted due to limited space).

Note from (15) that the contribution of each sensor in minimizing the MMSE Kalman estimation error is independent of other sensors' contribution. Hence, in the case of sensor selection from a pool of only one sensor type $(T=1)$, the problem becomes simply an ordering problem, i.e., the $\left\lfloor\lambda / \beta_{1}\right\rfloor$ sensors with maximum contribution, $\psi_{n, t}$, are selected where

$$
\psi_{n, t}=\frac{c_{n}^{2}}{\sigma_{\nu}^{2}} \cdot\left[1-\frac{\sigma_{u}^{2}}{g_{n}^{2}\left(\boldsymbol{w}_{n}^{T}\right) \sigma_{\nu}^{2}+\sigma_{u}^{2}}\right]
$$




\section{B. Greedy Algorithm Solution}

Since the contribution of each selected sensor is independent of other selected sensors, the problem might look similar to the $0-1$ knapsack problem. The problems are similar considering the analogy between the sensor contribution, $\psi_{n, t}$, and cost, $\beta_{t}$, to the item value and weight/size in the $0-1$ knapsack problem respectively. However, the sensor selection problem is different in that only one sensor at most can be placed at any sensing location.

The sensor types are ordered such that $\beta_{1}<\beta_{2}<\cdots<\beta_{T}$. To simplify the problem, we assume that $\beta_{t 2} / \beta_{t 1} \in \mathbb{Z}, \forall t_{2}>$ $t_{1}$. An auxiliary sensor type, $t=0$ is defined such that $\beta_{0}=$ $\eta_{0}=0$. By solving Algorithm 1, the solution is obtained in a sensor selection vector $\mathbf{b} \in \mathbb{R}^{N}$ such that the element $b_{i} \in$ $\{0,1, \ldots, T\}$ represents the sensor type at location $i$.

First, all the system budget, $\lambda$, is consumed by placing sensors of type 1 at optimal locations as discussed in (17) for the case of one sensor type (step 3). Then, we consider the selection of the next sensor type at each stage from 2 to $T$.

At the $t$-th stage, sensors of the $t$-th type are considered to replace a subset of the selected sensors which are of types $\{1, \ldots, t-1\}$. This is done by checking the performance improvement, denoted by $\phi_{i}$, of replacing the sensor at the $i$-th sensing location, with $\psi_{i, b_{i}}$, with the $t$-th sensor type, with a contribution $\psi_{i, t}$ and removing the least contributing subset of the selected sensors with contribution $\theta_{i}$ and budget $\beta_{t}-\beta_{b_{i}}$ (step 9). At each cycle, the replacement is made only for the sensor location at which the maximum performance improvement, $\phi_{\max }$, is achieved if $\phi_{\max }>0$. The replacement is made in step 12 in Algorithm 1. Note that each sensor of the least contributing subset is downgraded by one type level. The extra system cost caused by the replacement in step 12 is compensated by removing the least contributing sensors as in steps 13 to 16.

\section{Numerical EXPERIMENTS}

In this section, the discussed solutions in Section IV are validated by conducting an exemplary simulation. Consider a scalar state parameter, 100 candidate sensing location, fusion center and cellular base station, which is assumed to be the energy supplier to the selected sensors, located as shown in Figure 1.

The state parameter is modeled as in (1) with $a=.71$ and $\omega[k] \sim \mathcal{N}(0,1)$, hence, $\sigma_{x}^{2}=2$. The state parameter is then observed at the sensor level as in (2) such that $\sigma_{\nu}^{2}=1$ and $c_{n}[k]=\gamma_{1} \exp \left(\frac{d_{n}}{\gamma_{2}}\right)$, where $\gamma_{1}=10$ and $\gamma_{2}=-1 / 3$ are the source diffusion parameters and $d_{n}$ is the distance between the state parameter and the $n$-th sensing location. The system designer has the option to choose a sensor type from a pool of 3 sensor types with EH efficiency vector, $\boldsymbol{\eta}=\left[\begin{array}{lll}0.2 & 0.4 & 0.8\end{array}\right]^{T}$ and budget vector $\boldsymbol{\beta}=\left[\begin{array}{lll}1 & 2 & 4\end{array}\right]^{T}$. The sensor transmission power is modeled as $f\left(\rho_{n}, \boldsymbol{w}_{n}^{T} \boldsymbol{\eta}\right)=\min \left(\rho_{n} \boldsymbol{w}_{n}^{T} \boldsymbol{\eta}, 10 \boldsymbol{w}_{n}^{T} \boldsymbol{\eta}\right)$, such that $\rho_{n}=l_{n}^{-2}$ where $l$ is the distance between the energy supplier, i.e., the cellular base station, and the $n$-th sensing location. Finally, the observations are sent to the fusion center

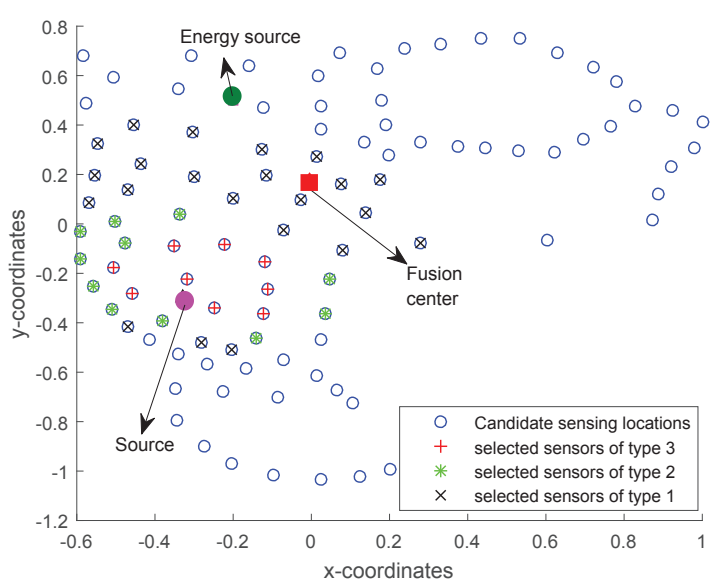

Fig. 1. Selected sensors

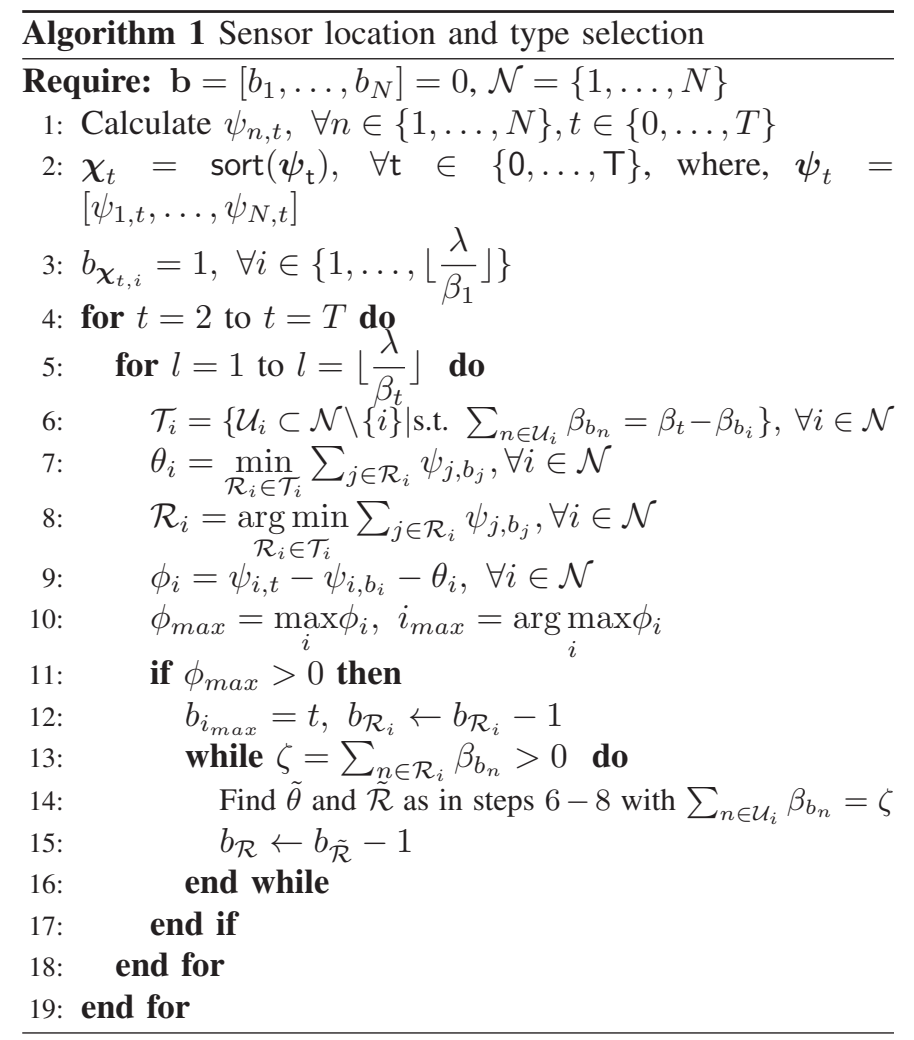

through an AWGN channel with receiver noise variance $\sigma_{u}^{2}=1$ and channel gain $h_{n}^{2}=r_{n}^{-2}$ where $r_{n}$ is the distance between the $n$-th sensing location and the fusion center.

Using Algorithm 1, the selected sensors for a maximum system budget of $\lambda=80$ are shown in Figure 1. By testing the solution for different system parameters, it is found that the lower the communication channel SNR, relative to the observation accuracy, the closer the selected sensors are to the fusion center and the energy supplier. Conversely, the sensors are centralized around the source location for relatively high channel SNR. In general, the higher the observation accuracy and the channel SNR at a sensing location, the higher the 


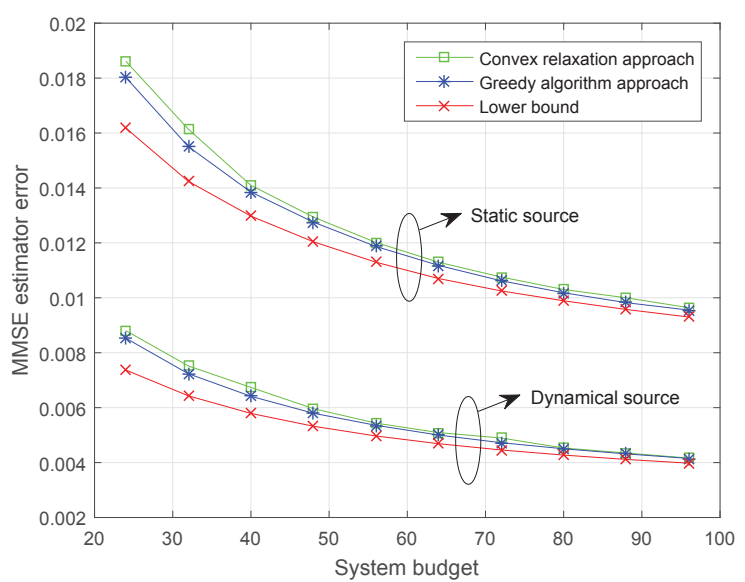

Fig. 2. Comparison between the obtained MMSE error using the convex relaxation approach and the greedy algorithm approach

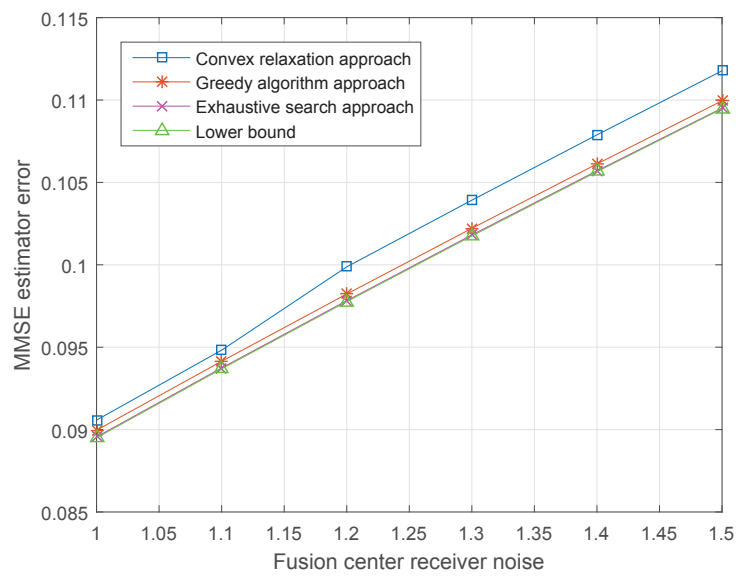

Fig. 3. The obtained MMSE error using the convex relaxation approach ,the greedy algorithm approach and the exhaustive search for a small system

sensor's power rating placed at that location.

As illustrated in Figure 2, we compare the performance of the convex relaxation approach and the greedy algorithm approach for different system budgets. We also show the obtained MMSE error in the case of static source, i.e., ignoring the temporal correlation. Figure 2 further illustrates a lower bound system performance which is obtained by solving the convex problem in (16) without rounding the solution. Note that this lower bound is, in general, unachievable since the solution of (16) is not binary in general. Finally to check the optimality of Algorithm 1, a small system with two types and 16 sensor nodes was simulated. Figure 3 shows the obtained MMSE error using the convex relaxation approach, the greedy algorithm approach and the exhaustive search versus the fusion center receiver noise.

\section{CONCLUSiON}

The sensor selection for scalar state parameter estimation in a WSN was studies in the paper. The dynamics of the state parameter at the source, the observation quality and available power at the sensors and the AWGN channel quality were taken into account in deriving the system mathematical model. In addition to the sensor placement, the power rating of the sensor is selected from a pool of available sensor types. Two sensor selection approaches were presented; convex relaxation approach and greedy algorithm approach. A numerical experiment was made to validate the obtained solutions. The results show the superiority of the greedy algorithm over the convex relaxation approach.

\section{REFERENCES}

[1] M. Shamaiah, S. Banerjee, and H. Vikalo, "Greedy sensor selection: Leveraging submodularity," in Decision and Control (CDC), 2010 49th IEEE Conference on. IEEE, 2010, pp. 2572-2577.

[2] S. Joshi and S. Boyd, "Sensor selection via convex optimization," IEEE Transactions on Signal Processing, vol. 57, no. 2, pp. 451-462, 2009.

[3] S. P. Chepuri and G. Leus, "Sparsity-promoting sensor selection for nonlinear measurement models," IEEE Transactions on Signal Processing, vol. 63, no. 3, pp. 684-698, 2015.

[4] M. Calvo-Fullana, J. Matamoros, C. Antón-Haro, and S. M. Fosson, "Sparsity-promoting sensor selection with energy harvesting constraints," in Acoustics, Speech and Signal Processing (ICASSP), 2016 IEEE International Conference on. IEEE, 2016, pp. 3766-3770.

[5] H. Zhang, R. Ayoub, and S. Sundaram, "Sensor selection for optimal filtering of linear dynamical systems: Complexity and approximation," in IEEE Conference on Decision and Control (CDC), 2015.

[6] _ "Sensor selection for kalman filtering of linear dynamical systems: Complexity, limitations and greedy algorithms," Automatica, 2017.

[7] E. J. Msechu and G. B. Giannakis, "Sensor-centric data reduction for estimation with wsns via censoring and quantization," IEEE Transactions on Signal Processing, vol. 60, no. 1, pp. 400-414, 2012.

[8] S. T. Jawaid and S. L. Smith, "Submodularity and greedy algorithms in sensor scheduling for linear dynamical systems," Automatica, vol. 61, pp. 282-288, 2015.

[9] C. O. Savage and B. F. La Scala, "Optimal scheduling of scalar gaussmarkov systems with a terminal cost function," IEEE Transactions on Automatic Control, vol. 54, no. 5, pp. 1100-1105, 2009.

[10] G. Wang, J. Chen, and J. Sun, "On sequential kalman filtering with scheduled measurements," in Cyber Technology in Automation, Control and Intelligent Systems (CYBER), 2013 IEEE 3rd Annual International Conference on. IEEE, 2013, pp. 450-455.

[11] M. Calvo-Fullana, J. Matamoros, and C. Antón-Haro, "Sensor selection and power allocation strategies for energy harvesting wireless sensor networks," IEEE Journal on Selected Areas in Communications, vol. 34, no. 12 , pp. $3685-3695,2016$.

[12] R. E. Kalman, "A New Approach to Linear Filtering and Prediction Problems," Transactions of the ASME Journal of Basic Engineering, no. 82 (Series D), pp. 35-45, 1960. [Online]. Available: http://www.cs.unc.edu/ welch/kalman/media/pdf/Kalman1960.pdf

[13] V. Tzoumas, A. Jadbabaie, and G. J. Pappas, "Sensor placement for optimal kalman filtering: Fundamental limits, submodularity, and algorithms," in IEEE American Control Conference (ACC), July 2016., 2015.

[14] T. Summers, "Actuator placement in networks using optimal control performance metrics," in Decision and Control (CDC), 2016 IEEE 55th Conference on. IEEE, 2016, pp. 2703-2708.

[15] Z. Ren, P. Cheng, J. Chen, L. Shi, and H. Zhang, "Dynamic sensor transmission power scheduling for remote state estimation," Automatica, vol. 50, no. 4, pp. 1235-1242, 2014.

[16] S. M. Kay, "Fundamentals of statistical signal processing: estimation theory," 1993.

[17] B. D. Anderson and J. B. Moore, "Optimal filtering," Englewood Cliffs, vol. 21 , pp. 22-95, 1979.

[18] S. Boyd and L. Vandenberghe, "Convex optimization," 2004. 